\title{
SELECTIVE DEPOSITION OF PARYLENE C FOR UNDERWATER SHEAR-STRESS SENSORS
}

\author{
Yong $\mathrm{Xu}^{(1)}, \mathrm{Yu}$-Chong Tai ${ }^{(2)}$ \\ ${ }^{(1)}$ Electrical and Computer Engineering, Wayne State University, Detroit, MI 48202, USA \\ ${ }^{(2)}$ Electrical Engineering, California Institute of Technology, Pasadena, CA 91125, USA
}

\begin{abstract}
This paper reports the application of selective parylene $C$ deposition for the waterproof coating of underwater shear-stress sensors. The selective deposition has been achieved by electrically heating the sensing element during deposition, utilizing the dependence of parylene deposition rate on substrate temperature. The electrical power supplied was only $20 \mathrm{~mW}$ to raise the temperature of the sensing element $210{ }^{\circ} \mathrm{C}$ above ambient due to the sensor's excellent thermal isolation. After selective parylene deposition, the sensing element was still exposed while other areas were covered by parylene. More effective interaction between the sensor and water was realized. With selective parylene deposition, excellent waterproof and high shear-stress sensitivity can be achieved at the same time.
\end{abstract}

\section{INTRODUCTION}

Parylene is the generic name for members of unique family of thermoplastic polymers that are vapor phase deposited by sublimating dimer of para-xylylene. The three most common types of parylene are referred to as: parylene $\mathrm{N}$, parylene $\mathrm{C}$, and parylene $\mathrm{D}$. Parylene has found numerous applications in micromachined transducers. Especially, it is used as protective coating against moistures, corrosives, and even body fluids because parylene exhibits excellent barrier properties [1]. In many cases, windows need to be opened to achieve a better interaction between transducers and environments. Usually, oxygen plasma is used to etch parylene and photoresist is chosen as mask material. The etch rates of parylene and photoresist in oxygen plasma are approximately same. This makes the patterning of thick parylene a troublesome process. Moreover, sometimes the parylene coating has to be carried out after packaging, in which case the conventional photolithography and oxygen plasma process is not applicable.

One example is the shear-stress sensor for underwater applications [2]. The structure of the sensor, which is schematically shown in Fig. 1 , is a polysilicon resistor seating atop a nitride diaphragm with a vacuum cavity underneath. During operation, the polysilicon resistor is heated electrically, while the fluid flowing over tends to cool it down. The input power of the sensor will change with the wall shear stress of the ambient flow field and this change can be readily detected electrically. The power is dissipated in two ways: conduction loss to substrate and convection loss to the fluid. The heat loss through radiation is negligible at the normal operation temperature. To increase the sensitivity and reduce the power consumption, we want most of the heat to be transferred to the fluid by convections. Namely, it is desirable to minimize the heat loss to substrate. This is achieved by the vacuum cavity underneath the polysilicon heater.

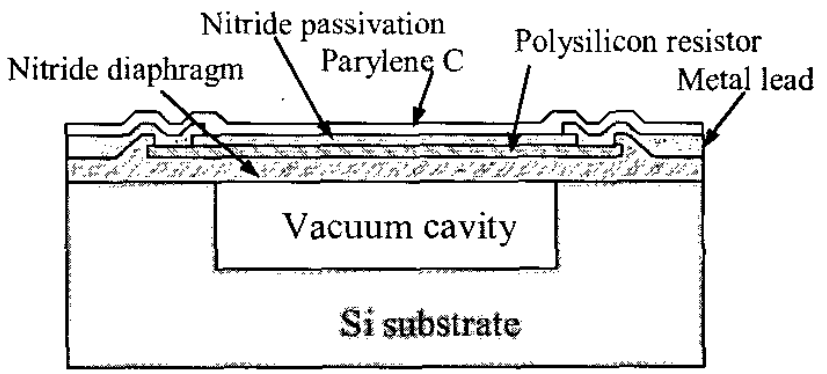

Figure 1. Cross section of the underwater shear-stress sensor uniformly coated with parylene C.

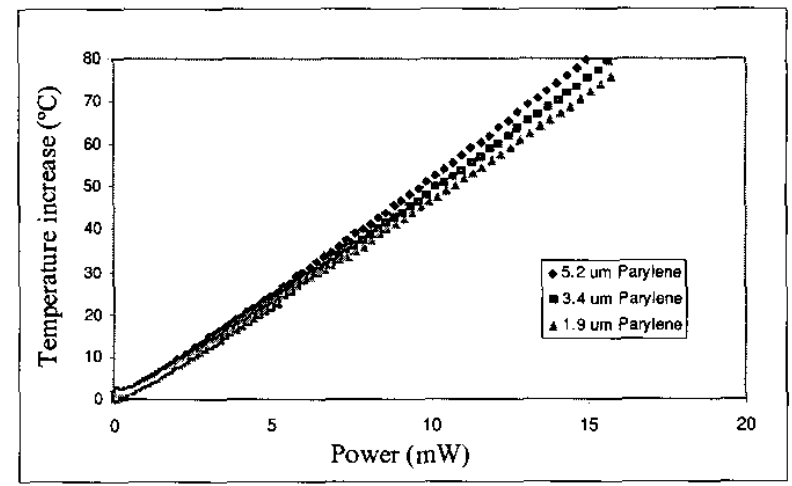

Figure 2. Temperature increase in water as a function of input power of one sensor coated with different thick parylene layers.

To operate under water, a waterproof layer needs to be deposited to encapsulate the metal wires. Parylene $\mathrm{C}$ is chosen as the waterproof material due to its small moisture transmission rate and its capability to be conveniently vapor phase deposited [2]. This waterproof 
layer (as shown in Fig. 1), on the other hand, limits the convective heat transfer from the sensor to water, resulting in the decrease of shear-stress sensitivity. Shown in the Fig. 2 is the temperature increase vs. power curves measured in water of the same sensor coated with different thick parylene $\mathrm{C}$ layers. The curves in Fig. 2 are derived from I-V measurements of the sensor at zero shear stress. This static thermal characteristic is very easy to obtain, but provides valuable information about the performance of the shear-stress sensor. It can be observed in Fig. 2 that as the parylene layer thickness increases, the curve shifts to the left. This means that less power is dissipated to water for thicker parylene layers, resulting in a lower sensitivity.

To achieve high sensitivity, the parylene layer should be as thin as possible. On the other hand, the parylene layer should be relatively thick for waterproof purpose. There seems to be a contradiction here. Nevertheless, if we observe the cross section of the sensor in Fig. 1 carefully, we can find out that the sensing element itself has already been encapsulated by silicon nitride, which is a superior barrier material. Therefore, there is no need to deposit parylene on the sensing element. Windows can be opened on the diaphragm to achieve a better thermal interaction between sensor and water. However, in this case we need to coat parylene after dicing and wire bonding the chip, which precludes the conventional photolithography and oxygen plasma etching process. Selective parylene deposition is employed here to expose sensing elements without using photolithography.

\section{EXPERIMENT}

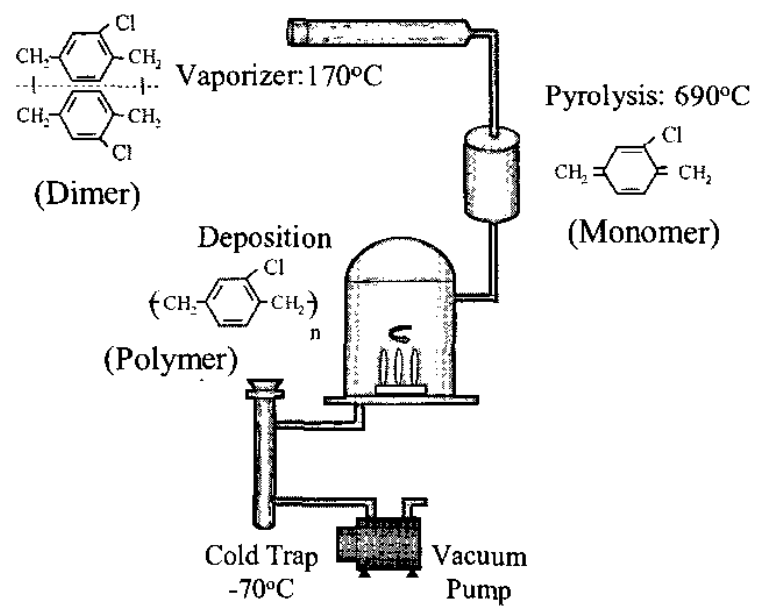

Figure 3. Simplified parylene deposition process. Adapted from [3].

The deposition process of parylene is illustrated in Fig. 3. First, solid dimer is vaporized at about $170{ }^{\circ} \mathrm{C}$ and then followed by the pyrolysis of the dimer at about $690{ }^{\circ} \mathrm{C}$ to yield monomer. Next, monomer enters the deposition chamber and deposits on any surfaces exposed. However, the deposition will only occur at surfaces where the temperature is below the ceiling temperature of polymerization. Therefore, the selective parylene deposition can be simply achieved by passing a current to the sensing element to electrically heat it during the deposition [4].

With a cavity underneath, only very small power is needed to heat up the shear-stress sensor as shown in Fig. 4. In the experiment, the input power is about $20 \mathrm{~mW}$ to achieve an average temperature increase of $210^{\circ} \mathrm{C}$ above ambient temperature. The $20 \mathrm{~mW}$ power can be easily provided by a battery. It is worth noting that the temperature distribution on the polysilicon resistor is not uniform. What Fig. 4 shows is the average temperature increase of the polysilicon resistor. Another issue we need to consider is the substrate temperature increase. If the substrate temperature is too high, no deposition will occur. A polysilicon temperature sensor is integrated on the substrate to monitor the temperature. As shown in Fig. 5, the temperature increase of the substrate is less than $3{ }^{\circ} \mathrm{C}$ after supplying electrical power to the sensor. Therefore, no substrate cooling is required during the deposition.

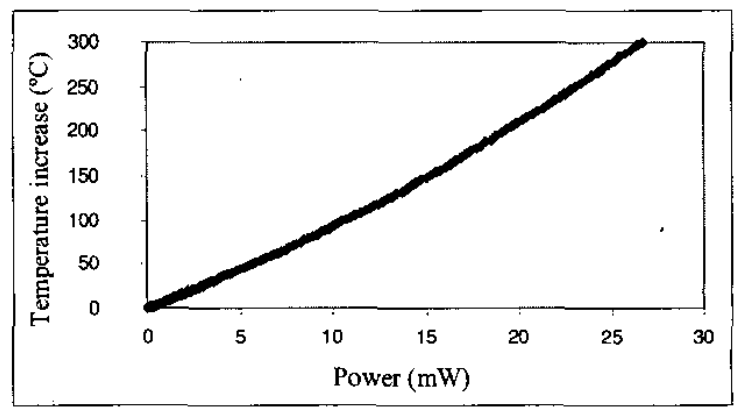

Figure 4. Temperature increase of the sensing element as a function of input power in vacuum.

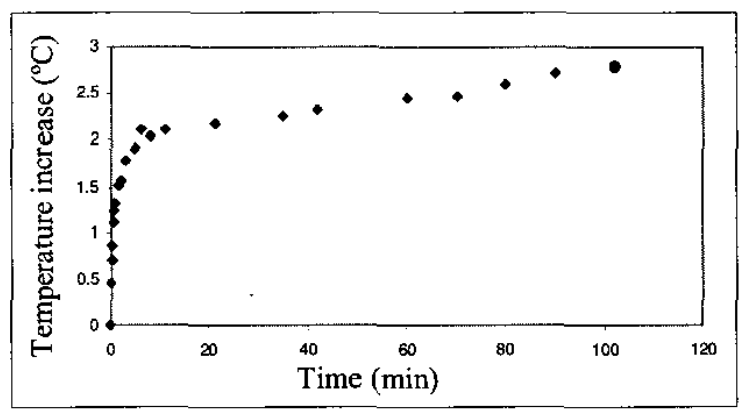

Figure 5. Temperature increase of the substrate in vacuum after turning on the power.

The selective parylene $\mathrm{C}$ deposition was carried out using a regular deposition system without any 
modification. A chip with multiple shear-stress sensors [2] was first attached to a printed circuits board, then wire bonded and soldered. Before loading this chip to the deposition chamber (Fig. 3), a battery was connected to one sensor on the chip to electrically heat it up. Other sensors on the chip were not powered and remained at room temperature. These sensors will be compared with the one selectively coated with parylene $\mathrm{C}$. The selective deposition of $5.2 \mu \mathrm{m}$ parylene $\mathrm{C}$ was finished within 3 hours.

\section{RESULTS AND DISCUSSIONS}

Figure 6 shows the picture of the sensor selectively coated with $5.2 \mu \mathrm{m}$ parylene $\mathrm{C}$ taken under a microscope. The interference rings caused by the thickness variation can be clearly observed. Very interestingly, these rings also represent the isothermal curves on the diaphragm, since parylene should have same thickness on isothermal curves. The isothermal curves on the diaphragm are difficult to obtain using infrared cameras due to the infrared radiation reflected back from the bottom of the cavity.

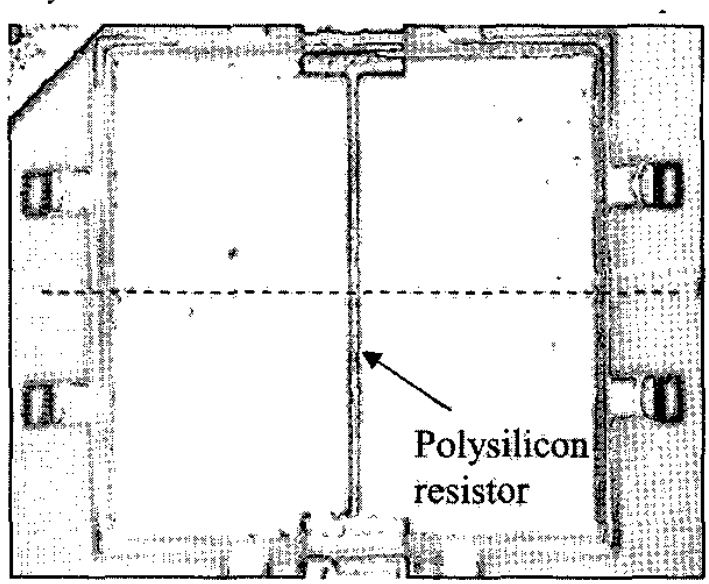

Figure 6. Micrograph of the shear-stress sensor selectively coated with $5.2 \mu \mathrm{m}$ parylene $C$. The diaphragm dimension is $210 \times 210 \mu \mathrm{m}^{2}$.

The surface profiles before and after $5.2 \mu \mathrm{m}$ selective parylene $\mathrm{C}$ deposition are shown in Fig. 7 (a) and (b). These curves were obtained by scanning along the center of the diaphragm (along the dashed line shown in Fig. 6) using a stylus surface profiler. In Figure 7 (a), the spike in the center is caused by the polysilicon resistor $(0.6 \mu \mathrm{m})$ while the two small spikes in the edge are due to the bird's beak of local oxidation [2]. Figure 7 (c) shows the thickness of parylene as a function of position after selectively depositing $5.2 \mu \mathrm{m}$ parylene $\mathrm{C}$ by subtracting (a) from (b). Outside the cavity, the parylene thickness is $5.2 \mu \mathrm{m}$. Starting from the edge of the cavity, the thickness decreases sharply.

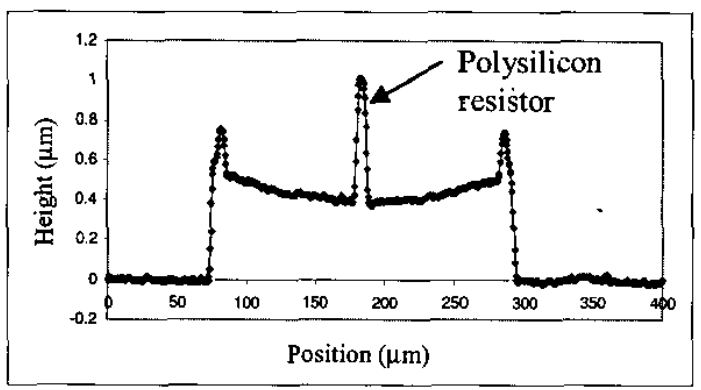

(a)

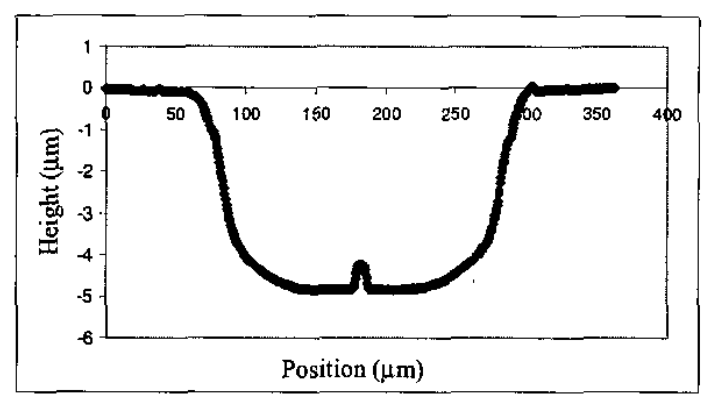

(b)

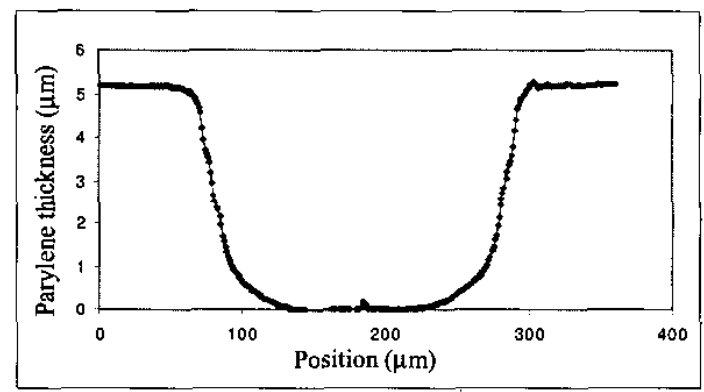

(c)

Figure 7. (a) Surface profile before parylene deposition; (b) surface profile after $5.2 \mu \mathrm{m}$ selective parylene deposition; (c) thickness of parylene across the center of the diaphragm.

At steady state, the temperature along the center of the diaphragm (the dashed line in Fig. 6) can be described by the following 1-D equation due to the symmetry:

$$
\frac{\partial^{2} T}{\partial x^{2}}=\frac{h}{k d}\left(T-T_{a}\right)
$$

where $h$ is the.convective heat transfer coefficient of ambient fluid, $k$ is the thermal conductivity of diaphragm material, $d$ is diaphragm thickness, and $T_{a}$ is the ambient temperature. Because the deposition is carried out in vacuum, the convection can be ignored $(h=0)$. Namely, the right side of the equation is equal to zero. Therefore, the temperature along the center of the diaphragm is a linear function of the position in vacuum. It is worth 
noting that thickness of parylene across the center of the diaphragm is not constant as shown in Fig. 7 (c). Nevertheless, the thickness of silicon nitride is $4 \mu \mathrm{m}$, comparable with the parylene layer, and the thermal conductivity of low stress silicon nitride $(3.2 \mathrm{~W} / \mathrm{m} \cdot \mathrm{K})$ is much larger than that of parylene $\mathrm{C}(0.082 \mathrm{~W} / \mathrm{m} \cdot \mathrm{K})$. The effect of the parylene layer on thermal conduction can be neglected to a first order approximation. The conclusion that the temperature along the center of the diaphragm is a linear function of the position is still valid. Based on this, we can derive the parylene thickness as a function of temperature from Fig. 7 (c). It can be estimated that when the temperature is above $140{ }^{\circ} \mathrm{C}$, the parylene $\mathrm{C}$ deposition rate is negligible.

To characterize the performance of the underwater shear-stress sensor, we can perform the shear-stress calibration in a well-characterized water tunnel. On the other hand, we can obtain the temperature vs. power relationship at zero shear stress easily by performing an $\mathrm{I}-\mathrm{V}$ curve measurement. As mentioned previously, this static thermal characteristic provides valuable information on the performance of the shear-stress sensor. Figure 8 compares the static thermal characteristics of two sensors, one uniformly coated with $5.2 \mu \mathrm{m}$ parylene $\mathrm{C}$ (S1) and the other selectively coated with $5.2 \mu \mathrm{m}$ parylene $C(S 2)$, in water and vacuum respectively.

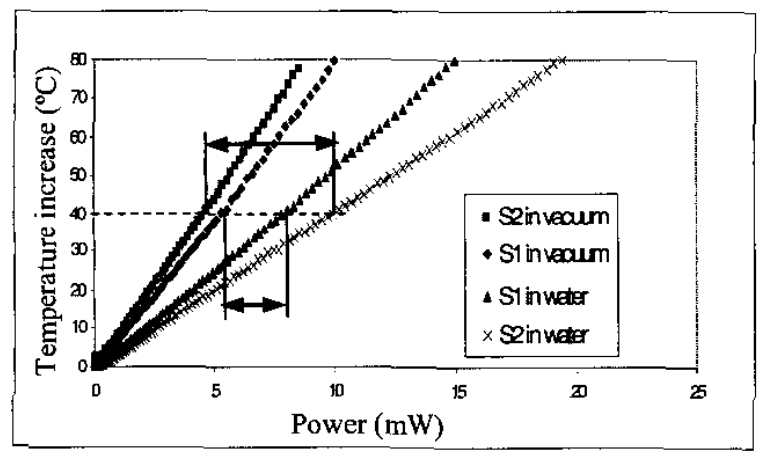

Figure 8. Temperature increase as a function of power consumption of two sensors ( $\mathrm{S} 1$ and $\mathrm{S} 2$ ) in vacuum and water respectively. S1: uniformly coated with $5.2 \mu \mathrm{m}$ parylene C; S2: selectively coated with $5.2 \mu \mathrm{m}$ parylene C.

The heat loss (power consumption) of the sensor consists of two parts: convective heat loss to ambient fluid $q_{1}$, and conductive heat loss to substrate through nitride and parylene thin films $q_{2}$. In vacuum, the convective heat loss can be neglected and the total heat lass is only the conductive heat loss $q_{2}$. When the sensor is immersed in water, the total heat loss is the sum of $q_{1}$ and $q_{2}$. To the first order approximation, $q_{2}$ can be considered as constants at the same temperature. Therefore, the heat convectively transferred to water $\left(q_{1}\right)$ can be estimated by the horizontal difference of the sensor's temperature-power curves measured in vacuum and water respectively. It can be observed in Fig. 8 that the sensor selectively coated with parylene C (S2) has more heat transferred to water than the sensor uniformly coated with parylene $\mathrm{C}$ (S1). For instance, with an operation temperature $40^{\circ} \mathrm{C}$ above ambient, $\mathrm{S} 2$ has about $5.3 \mathrm{~mW}$ power transferred to water while $\mathrm{S} 1$ has only about $2.6 \mathrm{~mW}$. Therefore, the sensor with selective parylene deposition has better thermal interaction with water and is expected to have higher sensitivity.

\section{CONCLUSIONS}

The selective parylene coating has been achieved simply by electrically heating the sensor during deposition. Due to the sensor's excellent thermal isolation, only very small power $(20 \mathrm{~mW})$ was needed and the substrate temperature increase was minimized $\left(<3{ }^{\circ} \mathrm{C}\right)$. The selective deposition process was carried out with a regular parylene coating system. We also obtained the isothermal curves on the sensor diaphragm. The ceiling temperature of parylene $\mathrm{C}$, above which the deposition rate is negligible, was estimated to be $140^{\circ} \mathrm{C}$. The temperature increase as a function of input power was also measured and compared for sensors selectively and uniformly coated with parylene $\mathrm{C}$. It is concluded that with selective parylene deposition, excellent waterproof and high shear-stress sensitivity can be achieved at the same time. The same method can also be employed in many other applications such as the packaging of some biosensors.

\section{ACKNOWLEDGMENTS}

This project was partly supported by the Office of Naval Research through an SBIR grant. The authors would also like to acknowledge the help of Mr. Hongyu Yu and Dr. Qiao Lin.

\section{REFERENCES}

[1] "Parylene Data Sheet," Specialty Coating System, Indianapolis.

[2] Y. Xu, F. Jiang, Q. Lin, J. Clendenen, S. Tung, and Y.-C. Tai, "Underwater Shear-Stress Sensor," presented at IEEE International Conference on Micro Electro Mechanical Systems (MEMS), Las Vegas, Nevada, 2002.

[3] X. Q. Wang, "Integrated Parylene Micro Electro Mechanical Systems," Ph.D. thesis, California Institute of Technology, 2000

[4] E. M. Charlson, E. J. Charlson, and R. Sabeti, "Temperature Selective Deposition of Parylene-C," IEEE Transactions on Biomedical Engineering, vol. 39 , pp. 202-206, 1992. 